\title{
ZERO-DISPERSION LIMIT FOR INTEGRABLE EQUATIONS ON THE HALF-LINE WITH LINEARISABLE DATA
}

\author{
A. S. FOKAS AND S. KAMVISSIS
}

Received 4 December 2002

We study the zero-dispersion limit for certain initial boundary value problems for the defocusing nonlinear Schrödinger (NLS) equation and for the Korteweg-de Vries (KdV) equation with dominant surface tension. These problems are formulated on the half-line and they involve linearisable boundary conditions.

\section{An initial boundary value problem for soliton equations}

In recent years, there has been a series of results of Fokas and collaborators on boundary value problems for soliton equations (see [3] for a comprehensive review). The method of Fokas in [3] goes beyond existence and uniqueness. In fact, it reduces these problems to Riemann-Hilbert factorisation problems in the complex plane, thus generalising the existing theory which reduces initial value problems to Riemann-Hilbert problems via the method of inverse scattering. One of the main advantages of the Riemann-Hilbert formulation is that one can use recent powerful results on the asymptotic behaviour of solutions to these problems (as some parameter goes to infinity) to derive asymptotics for the solution of the associated soliton equation. For the study of the long-time asymptotics, such methods were pioneered by Its and then made rigorous and systematic by Deift and Zhou; the method is known as "nonlinear steepest descent" in analogy with the linear steepest descent method which is applicable to asymptotic problems for Fouriertype integrals (see, e.g., [2]). A generalisation of the steepest descent method developed in [1] is able to give rigorous results for the so-called "semiclassical" or "zero-dispersion" limit of the solution of the Cauchy problem for $(1+1)$-dimensional integrable evolution equations, in the case where the Lax operator is selfadjoint. The method has been further extended in [9] for the "nonselfadjoint" case.

In a recent paper [8], Kamvissis, by making use of the nonlinear steepest descent method, has studied the "zero-dispersion" limit of the initial boundary value problem for the $(1+1)$-dimensional, integrable, defocusing, nonlinear Schrödinger (NLS) equation on the half-line, for quite general initial and boundary data. In this paper, we consider 
the simplest case of "linearisable" data. More precisely, we consider the two "archetypal" soliton equations

$$
\begin{gathered}
i h u_{t}(x, t)+h^{2} u_{x x}(x, t)-2|u(x, t)|^{2} u(x, t)=0, \quad x \geq 0, t \geq 0, \\
u(x, 0)=u_{0}(x) \in \mathbb{S}\left(\mathbb{R}^{+}\right), \quad 0<x<\infty
\end{gathered}
$$

with the linearisable boundary condition

$$
u_{x}(0, t)-\chi u(0, t)=0, \quad t>0
$$

for some constant $\chi \geq 0$, where $h$ is the semiclassical parameter which is assumed to be small and positive and $\mathbb{S}\left(\mathbb{R}^{+}\right)$denotes the Schwartz class on $[0, \infty)$;

$$
\begin{gathered}
u_{t}(x, t)+u_{x}(x, t)+6 u u_{x}(x, t)-h^{2} u_{x x x}(x, t)=0, \quad x \geq 0, t \geq 0, \\
u(x, 0)=u_{0}(x) \in \mathbb{S}\left(\mathbb{R}^{+}\right), \quad 0<x<\infty
\end{gathered}
$$

with the linearisable boundary condition

$$
u(0, t)=\chi, \quad u_{x x}(0, t)=\chi+3 \chi^{2}, t \geq 0
$$

for some constant $\chi$, where $h$ is the dispersion parameter which is assumed to be small and positive.

It is well known that these equations admit a "Lax-pair" formulation. Namely, these equations are the compatibility condition for the equations $L \mu=0$ and $B \mu=0$, where $L$ and $B$ are differential operators on a Hilbert space. In the NLS equation case, for example, they are given by

$$
\begin{gathered}
L=\left(\begin{array}{cc}
\partial_{x}-i k & i u \\
-i \bar{u} & \partial_{x}+i k
\end{array}\right), \\
B=\left(\begin{array}{cc}
i h \partial_{t}+4 i k^{2}+i|u|^{2} & -2 k u-i u_{t} \\
-2 k \bar{u}+i \bar{u}_{t} & i h \partial_{t}-i|u|^{2}
\end{array}\right) .
\end{gathered}
$$

Here, the bar denotes complex conjugation, $k$ is the spectral variable, and $u=u(x, t)$ is the solution of (1.1).

The traditional method of solving initial value problems for soliton equations that admit a Lax-pair formulation is to focus on the operator $L$ and apply the theory of scattering and inverse scattering to this operator.

On the other hand, one of the main ideas of the method of Fokas is that for initial boundary value problems, the two operators $L$ and $B$ should be on an equal footing. The scattering transform should be applied to both operators simultaneously, while a so-called global relation has to be imposed on the data to ensure compatibility (see relation (2.6)). The global relation will ensure existence, uniqueness, and the validity of the RiemannHilbert formulation. 


\section{The Riemann-Hilbert problem}

As shown in [4], initial boundary value problems for integrable evolution PDEs can be reduced to a Riemann-Hilbert factorisation problem, under the special assumption that the so-called global relation, a condition on the given data, holds (see relation (2.6)).

Consider first the NLS equation with general boundary conditions. Namely, either $u(0, t), u_{x}(0, t)$, or a relation between $u(0, t)$ and $u_{x}(0, t)$ is given. The situation for Korteweg-de Vries (KdV) equation is similar where two boundary conditions are given.

Let $\Sigma$ be the contour $\mathbb{R} \cup i \mathbb{R}$ with the following orientation:

(i) the real axis is oriented from left to right,

(ii) the positive imaginary axis is oriented from infinity towards zero,

(iii) the negative imaginary axis is oriented from infinity towards zero.

We use the following convention: the +-side of an oriented contour is always to its left, according to the given orientation.

Letting $M_{+}$and $M_{-}$denote the limits of $M$ on $\Sigma$ from left and right, respectively, we define the Riemann-Hilbert factorisation problem

$$
M_{+}(x, t, k)=M_{-}(x, t, k) J(x, t, k)
$$

where

$$
\begin{gathered}
J(x, t, k)= \begin{cases}J_{4}^{-1}, & k \in \mathbb{R}^{+}, \\
J_{1}^{-1}, & k \in i \mathbb{R}^{+}, \\
J_{3}^{-1}, & k \in i \mathbb{R}^{-},\end{cases} \\
J_{2}=J_{3} J_{4}^{-1} J_{1}, \quad k \in \mathbb{R}^{-},
\end{gathered}
$$

with

$$
\begin{gathered}
J_{1}=\left(\begin{array}{cc}
1 & 0 \\
\Gamma(k) e^{2 i \Theta} & 1
\end{array}\right), \\
J_{3}=\left(\begin{array}{cc}
1 & -\bar{\Gamma}(\bar{k}) e^{-2 i \Theta} \\
0 & 1
\end{array}\right), \\
J_{4}=\left(\begin{array}{cc}
1 & -\gamma(k) e^{-2 i \Theta} \\
\bar{\gamma}(k) e^{2 i \Theta} & 1-|\gamma(k)|^{2}
\end{array}\right), \\
\Theta(x, t, k)=\frac{\theta}{h}, \quad \theta=k x+2 k^{2} t .
\end{gathered}
$$

The functions $\gamma$ and $\Gamma$ are defined in terms of the spectral functions of the problem (see $[5,(2.25),(2.28)])$, with important analyticity properties (see $[5,(2.21),(2.22)])$. In particular,

$$
\Gamma(k)=\frac{1}{a(k)(a(k)(\bar{A}(\bar{k}) / \bar{B}(\bar{k}))-b(k))},
$$


where $a, b$ are the spectral functions for the $x$-problem and $A, B$ are the spectral functions for the $t$-problem. The functions $a, b$ are analytic and bounded in the upper half-plane, while $A, B$ are analytic and bounded in the first and third quadrants of the $k$-plane.

The solution of the NLS equation can be recovered from the solution of (2.1) as follows:

$$
u(x, t)=2 i h \lim _{k \rightarrow \infty}\left(k M^{12}(x, t, k)\right),
$$

where the index 12 denotes the (12)-entry of a matrix.

The following "global relation" is imposed on the scattering data:

$$
a(k) B(k)-b(k) A(k)=e^{4 i k^{2} T} c(k),
$$

where $c(k)$ is analytic and bounded for $\operatorname{Im} k>0$, and $c(k)=O(1 / k)$ as $k \rightarrow \infty$. Here, $T$ is the time up to which we solve the initial boundary value problem for NLS. In general, $A$, $B$ are functions of $T$.

There exists a complicated relation between $u(0, t)$ and $u_{x}(0, t)$; the global relation is the expression of this in the spectral space.

In our particular case (problem (1.1)), $T=\infty$ and the global relation becomes

$$
a(k) B(k)-b(k) A(k)=0
$$

for $\arg (k) \in[0, \pi / 2]$.

The KdV is treated similarly. The contour $\Sigma^{\mathrm{KdV}}$ consists of the real line oriented from left to right, together with the curves

$$
\begin{aligned}
& l_{+}=\left\{k=k_{R}+i k_{I}, k_{I}>0, \frac{1}{4}+3 k_{R}^{2}-k_{I}^{2}=0\right\}, \\
& l_{-}=\left\{k=k_{R}+i k_{I}, k_{I}<0, \frac{1}{4}+3 k_{R}^{2}-k_{I}^{2}=0\right\}
\end{aligned}
$$

oriented from right to left. Instead of (2.1), the Riemann-Hilbert problem becomes

$$
M_{+}^{\mathrm{KdV}}(x, t, k)=M_{-}^{\mathrm{KdV}}(x, t, k) J^{\mathrm{KdV}}(x, t, k),
$$

where

$$
\begin{aligned}
& J^{\mathrm{KdV}}(x, t, k)= \begin{cases}\left(J_{1}^{\mathrm{KdV}}\right)^{-1}, & k \in l_{-}, \\
\left(J_{3}^{\mathrm{KdV}}\right)^{-1}, & k \in l_{+},\end{cases} \\
& J_{2}^{\mathrm{KdV}}=J_{3}^{\mathrm{KdV}}\left(J_{4}^{\mathrm{KdV}}\right)^{-1} J_{1}^{\mathrm{KdV},}, \quad k \in \mathbb{R},
\end{aligned}
$$


with

$$
\begin{aligned}
& J_{1}^{\mathrm{KdV}}=\left(\begin{array}{cc}
1 & 0 \\
\Gamma(k) e^{2 i \Theta^{\mathrm{KdV}}} & 1
\end{array}\right), \\
& J_{3}^{\mathrm{KdV}}=\left(\begin{array}{cc}
1 & -\bar{\Gamma}(\bar{k}) e^{-2 i \Theta^{\mathrm{KdV}}} \\
0 & 1
\end{array}\right) \text {, } \\
& J_{4}^{\mathrm{KdV}}=\left(\begin{array}{cc}
1 & -\gamma(k) e^{-2 i \Theta^{\mathrm{KdV}}} \\
\bar{\gamma}(k) e^{2 i \Theta^{\mathrm{KdV}}} & 1-|\gamma(k)|^{2}
\end{array}\right), \\
& \Theta^{\mathrm{KdV}}(x, t, k)=\frac{\theta^{\mathrm{KdV}}}{h}, \quad \theta^{\mathrm{KdV}}=-k x+\left(k+4 k^{3}\right) t .
\end{aligned}
$$

Here, $\Gamma$ is still defined via (2.4) and the spectral functions $a, b, A$, and $B$ still satisfy (2.7), where of course these functions are now expressed in terms of the KdV spectral problems.

\section{Linearisable data}

In general, the global relation together with the definition of $A(k)$ and $B(k)$ imply a nonlinear Volterra integral equation for the missing boundary values. For example, it is shown in [5] that in the case of the defocusing NLS equation with $q(0, t)=f_{0}(t)$ given, the unknown boundary value $q_{x}(0, t)=f_{1}(t)$ satisfies a nonlinear Volterra equation which has a global solution.

We note that the analogous step for linear evolution equations is solved by algebraic manipulations [3]. This is a consequence of the invariance of the unknown terms in the global relation under $k \rightarrow \nu(k)$, where $\omega(\nu(k))=\omega(k)$ and $\omega$ is $k^{2}$ and $k+4 k^{3}$ for the NLS and $\mathrm{KdV}$, respectively. Unfortunately, the global relation now involves the solution of the $t$-problem $\Phi(t, k)$ which in general breaks the invariance. However, for a particular class of boundary conditions, this invariance survives. This is precisely the class of "linearisable problems," namely a class of problems for which $A(k)$ and $B(k)$ can be explicitly written in terms of $a(k)$ and $b(k)$.

It is shown in [4] that for the NLS equation with the boundary condition (1.2),

$$
\frac{B(k)}{A(k)}=-\frac{2 k+i \chi}{2 k-i \chi} \frac{b(-k)}{a(-k)},
$$

while for the KdV with the boundary condition (1.4),

$$
\frac{B(k)}{A(k)}=\frac{f(k) b(\nu(k))-a(\nu(k))}{f(k) a(\nu(k))-b(\nu(k))},
$$

where

$$
\begin{gathered}
v^{2}+k v+k^{2}+\frac{1}{4}=0, \\
f(k)=\frac{v+k}{v-k}\left(1-\frac{4 v k}{\chi}\right) .
\end{gathered}
$$


Now we restrict ourselves to NLS equation first. It is then easy to see that $B / A$ is analytic and $O(1 / k)$ in the first quadrant. Hence, the coefficient $\Gamma$ is analytic (at least) in the first quadrant of the $k$-plane, and bounded there.

This has an important consequence. Noting the decay properties of the term $\exp (2 i \Theta)$ as $k \rightarrow \infty$ in the first quadrant, it is immediate that the positive imaginary axis of the contour can be deformed clockwise to the positive real part. This deformation is exact, not approximate.

Similarly, the negative imaginary part of the contour can be deformed to the negative real axis. We end up with a Riemann-Hilbert problem with jumps only along the real axis. In fact, let

$$
\begin{aligned}
& N(x, t, k)=M(x, t, k), \quad \arg (k) \in\left(\frac{\pi}{2}, \frac{3 \pi}{2}\right), \\
& N(x, t, k)=M(x, t, k) J_{1}^{-1}, \quad \arg (k) \in\left(0, \frac{\pi}{2}\right), \\
& N(x, t, k)=M(x, t, k) J_{3}^{-1}, \quad \arg (k) \in\left(\frac{3 \pi}{2}, 2 \pi\right) .
\end{aligned}
$$

The Riemann-Hilbert problem becomes

$$
\begin{gathered}
N_{+}(x, t, k)=N_{-}(x, t, k) J(x, t, k), \quad k \in \mathbb{R}, \quad \lim _{k \rightarrow \infty} N(x, t, k)=I, \\
\text { where } J_{N}(x, t, k)=\left(\begin{array}{cc}
1 & (\gamma-\bar{\Gamma})(k) e^{-2 i \Theta} \\
-(\bar{\gamma}-\Gamma)(k) e^{2 i \Theta} & 1-|\gamma(k)|^{2}
\end{array}\right),
\end{gathered}
$$

and formula (2.5) holds with $N$ instead of $M$. Again, we stress that problems (2.1) and (3.5) are exactly equivalent, not just approximately equivalent.

Note that for $k \geq 0, \gamma(k)-\bar{\Gamma}(k)=0$. This follows from the definition in (2.4) and the global relation (2.6). For negative $k, \gamma(k)-\bar{\Gamma}(k)=(b A-a B) /(\bar{a} A-\bar{b} B)$ has all the smoothness and decay properties that are required from a bona fide reflection coefficient corresponding to a realised potential. For example, if the initial data $u_{0}$ belongs to the Schwartz class of $\mathbb{R}^{+}$, then $a$ and $b$ are also Schwartz, while $A$ and $B$ are smooth and bounded. Hence, $R=\gamma-\bar{\Gamma}$ belongs to the Schwartz class of $\mathbb{R}$. There is a unique potential $v_{0}$ corresponding to $R$, which is a continuation of $u_{0}$. The Riemann-Hilbert problem (3.5) then gives the evolution of the solution to the NLS equation under initial data $v_{0}$.

Thus, we have shown that, in the linearisable case for NLS equation, the half-line problem can be recovered from the solution of the full real line problem by appropriately continuing the initial data.

The above observations are an immediate consequence of the question of studying the semiclassical limit of NLS equation. Since the initial boundary value problem can be considered as a restriction of an initial value problem and since the initial value problem for NLS equation is well understood (studied by Jin et al. in $[6,7]$ ), the results for the initial boundary value problem are recovered immediately. 
More precisely, with the introduction of a small dispersive constant $h$, the changes in the Riemann-Hilbert problem (2.1) will be as follows:

(i) $x, t$ will be replaced by $x / h, t / h$;

(ii) the coefficients $\gamma, \Gamma$ will now be dependent on $h$.

Since the deformation leading to (3.5) is exact, that is, there is no error (possibly dependent on $h$ ), the reduction to a Riemann-Hilbert problem on the line is possible, exactly as in the $h=1$ case.

Phenomenologically, one sees that the half-plane $x, t \geq 0$ can be divided into two regions. In the first "smooth" region, strong semiclassical limits exist and satisfy the formally limiting system. In the NLS equation case, letting $\rho=|u|^{2}, \mu=h \operatorname{Im}\left(\bar{u} u_{x}\right)$, the strong limits $\tilde{\rho}, \tilde{\mu}$, as $h \rightarrow 0$, exist and satisfy

$$
\tilde{\rho}_{t}+\tilde{\mu}_{x}=0, \quad \tilde{\mu}_{t}+\left(\frac{\tilde{\mu}^{2}}{\tilde{\rho}}-\frac{\tilde{\rho}^{2}}{2}\right)_{x}=0 .
$$

In the second "turbulent" region, fast oscillations appear that can be described in terms of slowly modulating finite-gap solutions. Only weak limits exist for $\rho, \mu$ as $h \rightarrow 0$ and they can be expressed in terms of the solutions of the so-called Whitham system. Rigorous asymptotic formulae for $\rho, \mu$ are also easily available (see, e.g., [9]).

In the case of the KdV equation, even with the appropriate changes of contour and phase, the situation is more complicated. It is not true anymore, even for linearisable data, that the half-line problem can be recovered from the solution of the full real line problem by appropriately continuing the initial data, for any value of the small dispersion parameter $h$. However, we will show that this reduction is possible asymptotically as $h \rightarrow 0$.

Denote by $D_{1}$ and $D_{2}$ the domains bounded by $l_{+} \cup \mathbb{R}$ and $l_{-} \cup \mathbb{R}$, respectively. In order to follow the argument used above for the NLS equation, we need to "deform" the curves $l_{-}$and $l_{+}$to the real line. This is not possible because $\bar{\Gamma}(\bar{k}) e^{-2 i \Theta^{\mathrm{KdV}}}$ and $\Gamma(k) e^{2 i \Theta^{\mathrm{KdV}}}$ are not bounded as $k \rightarrow \infty$ in the domains $D_{1}$ and $D_{2}$, respectively. What we can do is to "conjugate away" the jumps on $l_{-}$and $l_{+}$by introducing an auxiliary Riemann-Hilbert problem which can be reduced to a scalar (hence explicitly solvable) Riemann-Hilbert problem. This is possible because of the triangularity of the jump matrices $J_{1}^{\mathrm{KdV}}$ and $J_{3}^{\mathrm{KdV}}$.

In the end, we still obtain a Riemann-Hilbert problem on the real line, which, however, is not of the "standard" KdV form, at least for general $h$. As long as we are only interested in the asymptotics $h \rightarrow 0$, though, the reduction to a "standard" Riemann-Hilbert problem is possible. This means that the already existing analysis of the zero-dispersion limit of $\mathrm{KdV}$ on the full line (see $[10,11,12,13])$ is after all applicable.

More precisely, following [8], we consider the following Riemann-Hilbert problem:

$$
L_{+}(x, t, k)=L_{-}(x, t, k) J^{\mathrm{KdV}}(x, t, k),
$$

where

$$
J^{\mathrm{KdV}}(x, t, k)=\left(J_{3}^{\mathrm{KdV}}\right)^{-1}, \quad k \in l_{+},
$$


368 Dispersionless integrable equations on the half-line

with

$$
J_{3}^{\mathrm{KdV}}=\left(\begin{array}{cc}
1 & -\bar{\Gamma}(\bar{k}) e^{-2 i \Theta^{\mathrm{KdV}}} \\
0 & 1
\end{array}\right), \quad \Theta^{\mathrm{KdV}}(x, t, k)=\frac{\theta^{\mathrm{KdV}}}{h}
$$

where

$$
\theta^{\mathrm{KdV}}=-k x+\left(k+4 k^{3}\right) t
$$

such that $\lim _{k \rightarrow \infty} L(x, t, k)=I$.

This problem can be solved explicitly as follows:

$$
L(x, t, k)=\left(\begin{array}{cc}
1 & l(x, t, k) \\
0 & 1
\end{array}\right)
$$

where

$$
l(x, t, k)=\frac{1}{2 \pi i} \int_{l^{+}} \frac{-\bar{\Gamma}(\bar{s}) e^{-2 i \Theta^{\mathrm{KdV}}(x, t, s)} d s}{s-k} .
$$

Similarly, consider

$$
U_{+}(x, t, k)=U_{-}(x, t, k)\left(J_{1}^{\mathrm{KdV}}\right)^{-1}(x, t, k), \quad k \in l_{-},
$$

with

$$
J_{1}^{\mathrm{KdV}}=\left(\begin{array}{cc}
1 & 0 \\
\Gamma(k) e^{2 i \Theta^{\mathrm{KdV}}} & 1
\end{array}\right)
$$

such that $\lim _{k \rightarrow \infty} U(x, t, k)=I$. We now have

$$
U(x, t, k)=\left(\begin{array}{cc}
1 & 0 \\
u(x, t, k) & 1
\end{array}\right)
$$

where

$$
u(x, t, k)=\frac{1}{2 \pi i} \int_{l^{-}} \frac{\Gamma(s) e^{2 i \Theta^{\mathrm{KdV}}(x, t, s)} d s}{s-k} .
$$

Now set

$$
N(x, t, k)= \begin{cases}M(x, t, k) U^{-1}(x, t, k), & k \in D_{1}, \\ M(x, t, k) L^{-1}(x, t, k), & k \in D_{2}, \\ M(x, t, k), & \text { otherwise. }\end{cases}
$$


Then $N(x, t, k)$ is analytic in $\mathbb{C} \backslash \mathbb{R}$, with $\lim _{k \rightarrow \infty} N(x, t, k)=I$, and across $\mathbb{R}$ the jump is given by

$$
N_{+}(x, t, k)=N_{-}(x, t, k) L(x, t, k) J(x, t, k) U^{-1}(x, t, k) .
$$

An easy deformation argument shows that $L=I+O(h), U=I+O(h)$ as $h \rightarrow 0$ (one can simply deform the contour "upwards" or "downwards" and the real part of the phase will become negative). This means that the factor $L$ can be ignored asymptotically. (This is not entirely obvious at this point. The full-line zero-dispersion analysis of $\mathrm{KdV}$ involves the introduction of a so-called $g$-function and a conjugation of the Riemann-Hilbert problem by a term $e^{\sigma_{3} g / h}$. Only after obtaining the new "conjugated" Riemann-Hilbert problem one is allowed to use the fact that $L, U=I+O(h)$ as $h \rightarrow 0$. See [8] for details.)

Again, the half-plane $x, t \geq 0$ can be divided into two regions. In the first "smooth" region, a strong zero-dispersion limit $\tilde{u}=\lim _{h \rightarrow 0} u$ exists and satisfies the formally limiting system

$$
\tilde{u}_{t}(x, t)+\tilde{u}_{x}(x, t)+6 \tilde{u} \tilde{u}_{x}(x, t)=0 .
$$

In the second "turbulent" region, fast oscillations appear that can be described in terms of slowly modulating finite-gap solutions. Only a weak limit exists for $u$ as $h \rightarrow 0$ and it can be expressed in terms of the solutions of the Whitham system for KdV. A rigorous asymptotic formula for $u$ is also easily available (see, e.g., [13]).

\section{References}

[1] P. Deift, S. Venakides, and X. Zhou, New results in small dispersion KdV by an extension of the steepest descent method for Riemann-Hilbert problems, Int. Math. Res. Not. 1997 (1997), no. 6, 286-299.

[2] P. A. Deift, A. R. Its, and X. Zhou, Long-time asymptotics for integrable nonlinear wave equations, Important Developments in Soliton Theory (A. S. Fokas and V. E. Zakharov, eds.), Springer Ser. Nonlinear Dynam., Springer-Verlag, Berlin, 1993, pp. 181-204.

[3] A. S. Fokas, On the integrability of linear and nonlinear partial differential equations, J. Math. Phys. 41 (2000), no. 6, 4188-4237.

[4] - Integrable nonlinear evolution equations on the half-line, Comm. Math. Phys. 230 (2002), no. 1, 1-39.

[5] A. S. Fokas, A. R. Its, and L.-Y. Sung, The nonlinear Schrödinger equation on the half-line, preprint, 2001.

[6] S. Jin, C. D. Levermore, and D. W. McLaughlin, The behavior of solutions of the NLS equation in the semiclassical limit, Singular Limits of Dispersive Waves (Lyon, 1991) (N. M. Ercolani, I. R. Gabitov, C. D. Levermore, and D. Serre, eds.), NATO Adv. Sci. Inst. Ser. B Phys., vol. 320, New York, 1994, pp. 235-255.

[7] The semiclassical limit of the defocusing NLS hierarchy, Comm. Pure Appl. Math. 52 (1999), no. 5, 613-654.

[8] S. Kamvissis, Semiclassical nonlinear Schrödinger on the half line, J. Math. Phys. 44 (2003), no. 12, 5849-5868.

[9] S. Kamvissis, K. D. T.-R. McLaughlin, and P. D. Miller, Semiclassical Soliton Ensembles for the Focusing Nonlinear Schrödinger Equation, Annals of Mathematics Studies, vol. 154, Princeton University Press, New Jersey, 2003. 
[10] P. D. Lax and C. D. Levermore, The small dispersion limit of the Korteweg-de Vries equation. I, Comm. Pure Appl. Math. 36 (1983), no. 3, 253-290.

[11] The small dispersion limit of the Korteweg-de Vries equation. II, Comm. Pure Appl. Math. 36 (1983), no. 5, 571-593.

[12] The small dispersion limit of the Korteweg-de Vries equation. III, Comm. Pure Appl. Math. 36 (1983), no. 6, 809-829.

[13] S. Venakides, The Korteweg-de Vries equation with small dispersion: higher order Lax-Levermore theory, Comm. Pure Appl. Math. 43 (1990), no. 3, 335-361.

A. S. Fokas: Department of Applied Mathematics and Theoretical Physics, University of Cambridge, Cambridge CB3 0WA, UK

E-mail address: t.fokas@damtp.cam.ac.uk

S. Kamvissis: Max-Planck-Institut für Mathematik, Vivatsgasse 7, Bonn 53111, Germany

E-mail address: spyros@mpim-bonn.mpg.de 


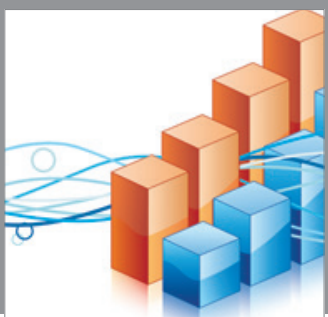

Advances in

Operations Research

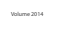

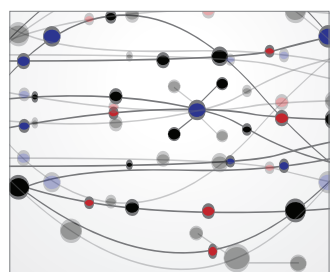

\section{The Scientific} World Journal
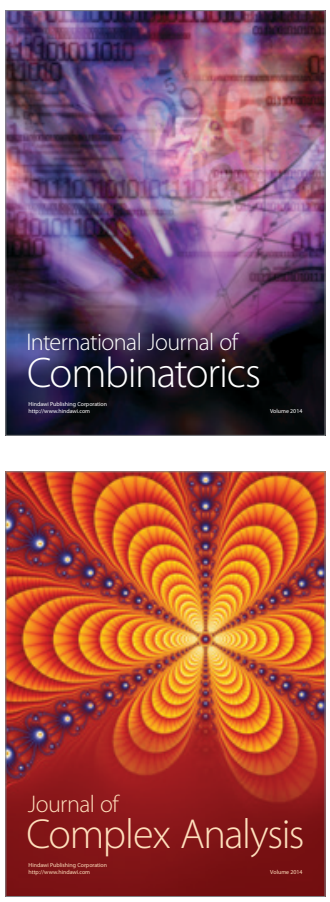

International Journal of

Mathematics and

Mathematical

Sciences
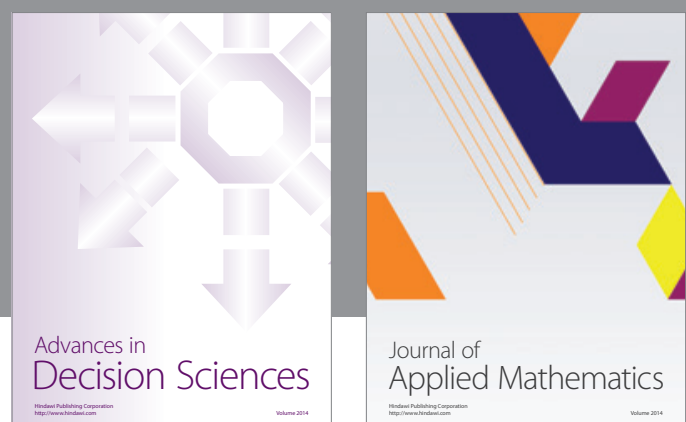

Journal of

Applied Mathematics
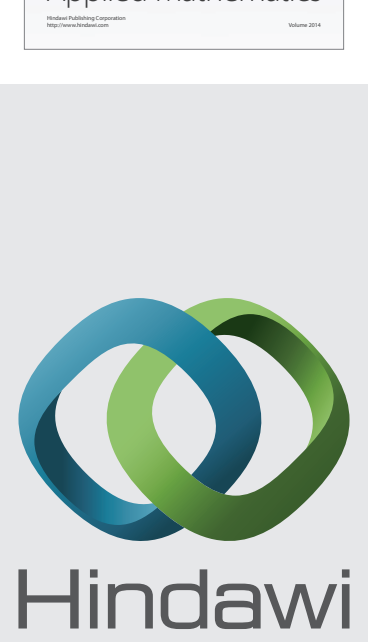

Submit your manuscripts at http://www.hindawi.com
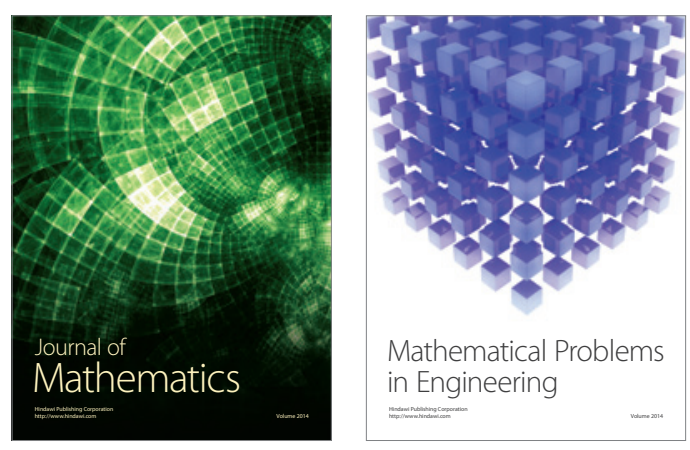

Mathematical Problems in Engineering
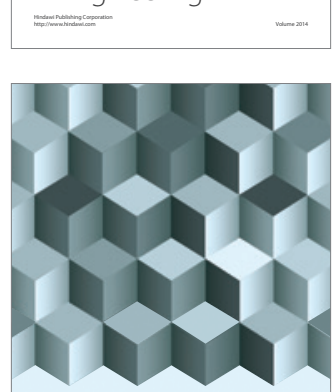

Journal of

Function Spaces
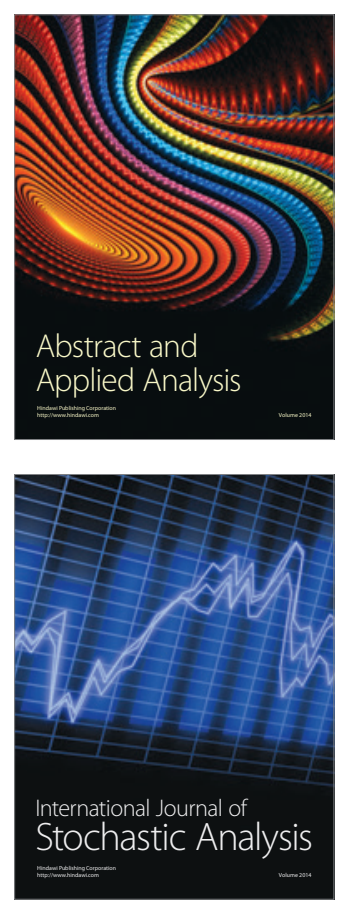

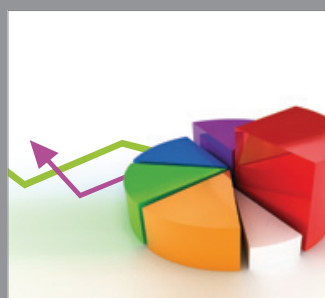

ournal of

Probability and Statistics

Promensencen
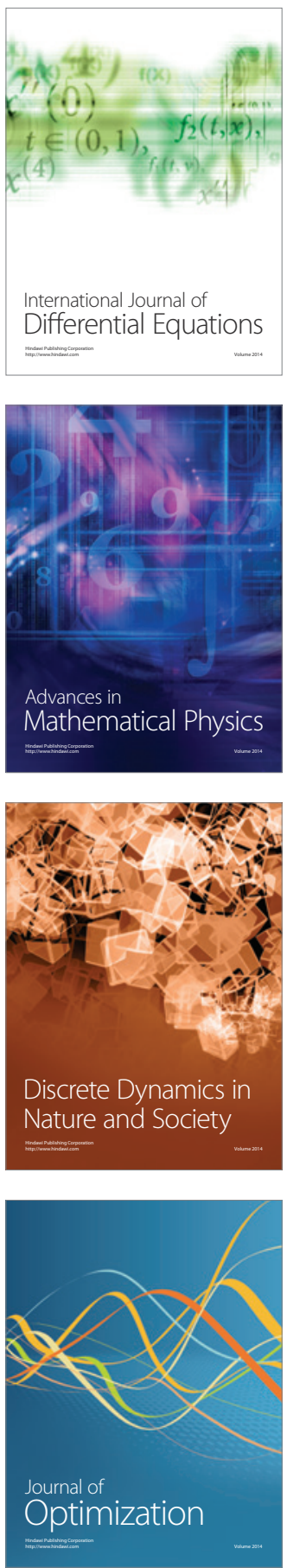